\title{
Wireless sensor networks: modeling and simulation
}

\author{
Sajjad A. Madani \\ COMSATS Institute of Information Technology, Abbottabad * \\ Pakistan \\ Jawad Kazmi \\ COMSATS Institute of Information Technology, Abbottabad ${ }^{\dagger}$ \\ Pakistan \\ Stefan Mahlknecht \\ Vienna University of Technology, Vienna $\ddagger$ \\ Austria
}

\begin{abstract}
Although different modeling techniques have been proposed during the last 300 years, the differential equation formalism proposed by Newton and Leibniz has been the tool of choice for modeling and problem solving Taylor (1996); Wainer (2009). Differential equations provide a formal mathematical method (sometimes also called an analytical method) for studying the entity of interest.

Computational methods based on differential equations could not be easily applied in studying human-made dynamic systems (e.g., traffic controllers, robotic arms, automated factories, production plants, computer networks, VLSI circuits). These systems are usually referred to as discrete event systems because their states do not change continuously but, rather, because of the occurrence of events. This makes them asynchronous, inherently concurrent, and highly nonlinear, rendering their modeling and simulation different from that used in traditional approaches. In order to improve the model definition for this class of systems, a number of techniques were introduced, including Petri Nets, Finite State Machines, min-max algebra, Timed Automata, etc. Banks \& Nicol. (2005); Cassandras (1993); Cellier \& Kofman. (2006); Fishwick (1995); Law \& Kelton (2000); Toffoli \& Margolus. (1987).

Wireless Sensor Network (WSN) is a discrete event system which consists of a network of sensor nodes equipped with sensing, computing, power, and communication modules to monitor certain phenomenon such as environmental data or object tracking Zhao \& Guibas (2004). Emerging applications of wireless sensor networks are comprised of asset and warehouse
\end{abstract}

\footnotetext{
*madanidcit.net.pk

†jawhaikaz@ciit.net.pk

$\ddagger_{\text {mahlknechteict.tuwien.ac.at }}$
} 
management, automotive, home and building automation, civil infrastructure monitoring, healthcare, industrial process control, military battlefield awareness, and security and surveillance Cerpa et al. (2001).

As discussed earlier, modeling and simulation is a mean to verify the working and to measure the effectiveness of the different techniques proposed for WSNs. Analytical modeling provides quick insight for the techniques developed for WSNs but fail to give realistic results because of WSN specific constraints like limited energy and sheer number of sensor nodes Chen et al. (2006). Real world implementation and test beds are the most accurate method to verify the concepts but are restricted by costs, effort, and time factors as well as repeating environmental conditions is also not possible Zeigler (1976). Simulations provide a good approximation to verify different schemes and applications developed for WSNs at low cost and in less time. To have credible results through simulation, the choice of models and the simulation environment is important.

There is always a tradeoff between credible simulation results and the time required to get these simulation results. The results always depend upon the level of abstraction of the models. The more detailed is the model, the better the accuracy of results but higher the amount of time required for simulation.

The models used for simulation can have a significant impact on the overall simulation study. In this chapter, we will present a brief overview of the models available for different modules of sensor network simulation study in addition to the general-purpose simulation frameworks and tools that can be used to study WSNs. Such tools include NS-2, OMNeT ++, SenSim, NesCT, GlomoSim, OPNET Modeler, SENSE, Ptolemy II, VisualSense and J-Sim. Additionally, some WSNs specific simulators frameworks/emulators are also covered including TOSSIM, EmStar, ATEMU and PAWiS.

\section{Introduction}

Recent advances in MEMS and distributed computing has enabled WSNs powered diverse applications ranging from military to kindergartens. WSN is a network of sensor nodes equipped with sensing, computing, power, and communication modules to monitor certain phenomenon such as environmental data or object tracking Raghavendra et al. (2004). In current scenarios, the number of sensor nodes may be 20 to 30 but in future it may consist of $n^{\text {th }}$ power more sensor and actuator systems Dietrich et al. (2001). The position of the sensor nodes may not be pre-determined and require sensor nodes to be equipped with self organizing protocols Akyildiz et al. (2002).Generally, sensor nodes observe and sense the phenomenon with a sensing module, process the data with a computing module, and send the data to a required destination via wireless link with a communication module.

\section{Wireless Sensor Network Applications}

Emerging applications of wireless sensor networks are comprised of asset and warehouse management, automotives, home and building automation, civil infrastructure monitoring, healthcare, industrial process control, military battlefield awareness, and security and surveillance Zhao \& Guibas (2004). Some examples of these application like robotic navigation $\mathrm{Fu}$ et al. (2009), aircraft corrosion monitoring Demo et al. (2010), direct load control in residential areas Molina-Garcia et al. (2007), personal mobile physiological monitoring and management system for chronic disease Toh et al. (2008), Wildfire detection Antoine-Santoni et al. (2006), 
in-service motor monitoring and energy management $\mathrm{Hu}$ (2008), application in petrochemical industry Ke et al. (2008), application for critical infrastructure risk analysis of fossil fuelled power stations Isreb (2006) and tactical military applications Lee et al. (2009) can be found in the litrature.

\section{Historical Perspective}

History of wireless communication used in the field can be traced back to late 1890's to AngloBoer war in Namibia, the then German Southwest Africa, and was declared by IEEE as electrical engineering milestone in which Marconi's system was used for wireless communication Sarkar et al. (2006). In 1921, the US Department of Defense initiated a radiotelegraphic network, which resulted in 125 stations network by 1925 Callaway et al. (2002). The Aloha system Abramson (1970) developed at the University of Hawaii is considered the first successful data network, which connected different campuses of the University of Hawaii. The Packet Radio Network Jubin \& Tornow (1987), comprising of 138 network devices was developed in 1972. In 1997, IEEE 802 LAN/MAN Standard Committee released the first Wireless LAN standard. The development of Wireless Personal Area Networks began in 1997, with the formation of Home RF Working Group and with the formation of Bluetooth Special Interest Group in 1998 Callaway et al. (2002). Like other wireless networks, the sensor networks also has a long history with can be traced back to 1978 DARPA -sponsored Distributed Sensor Nets Workshop. Wireless Integrated Network Sensors (WINS) ${ }^{1}$ project was initiated in 1993 by University of California at Los Angeles. The University of California at Berkeley started PicoRadio ${ }^{2}$ project in 1999. AMPS ${ }^{3}$ project started in MIT, to develop a complete architecture for low power wireless sensor networks. LonWorks Dietrich et al. (2001) in 2001 and EIB: Installation Bus System Sauter et al. (2002) in 2002 was a big step towards building automation. Wireless Self Sustaining Sensor Network Project ${ }^{4}$ (WSSN) at institute of computer technology, Vienna University of technology aimed to establish sensor networks for building automation which are low power and self sufficient in energy.

\section{Discret Event Modeling and Simulation Methodologies}

Modeling techniques for discrete event driven systems (including WSNs) are relatively recent. In this section, we present a non-comprehensive list of some of the formal modeling techniques created for modeling these systems.

\subsection{Automaton}

An automaton is defined as a graph representing system states and the transitions between them. The automaton receives a string of symbols as input, and it recognizes/rejects the inputs by advancing through the transitions. The input is read one symbol at a time; depending on the ending state, the automaton will accept or reject the input Cassandras (1993).

\subsection{Timed automata}

Timed automata, in particular, use clocks to describe the model's timing behavior Alur \& Dill (1994). The automaton is defined as a graph of states associated with clocks that determine

\footnotetext{
${ }^{1}$ www.janet.ucla.edu

${ }^{2}$ bwrc.eecs.berkeley.edu

${ }^{3}$ www-mtl.mit.edu

${ }^{4}$ http://www.ict.tuwien.ac.at/wireless
} 
the passage of time since the occurrence of an event. Every link is associated with a timing constraint that will define when the transition can be triggered. Whenever a transition executes, the associated clocks are reset. Timing constraints can also be associated with the model states, defining the duration of each of the states.

\subsection{Finite state machines}

Finite state machines (FSMs) can be represented as a graph in which the system's behavior is defined as a finite set of nodes (the model's states) and links between them (transitions between states). A given state reflects the evolution of the model, and transitions are associated with a given logical condition to enable the execution of the transition. When entering a state, an entry action can be executed (and an exit action can be executed when leaving it). Likewise, an input action can be triggered based on the current state and an input Cassandras (1993). An FSM is formally defined as

$$
F S M=(S, X, Y, f, g)
$$

Where

$X=$ finite input set

$\mathrm{Y}=$ finite output set

$\mathrm{S}=$ finite state set

\subsection{Markove chain}

A Markov chain is a discrete-time stochastic model described using a graph. Models' states are defined as nodes in the graph, and transitions between states are represented by links. One important property of Markov chains is that they are memoryless; thus, no state has a cause-effect relationship with the previous state. Therefore, knowledge of previous states is irrelevant for predicting the probability of the future states.

\subsection{Generalized Semi-Markovian Process}

A Generalized Semi-Markovian Process (GSMP) is a stochastic process (i.e., a collection of random variables over a probability space indexed by time). A GSMP is based on the notion of a state that makes a transition when an event associated with the current state occurs, and the state space is generated by a stochastic timed automaton Glynn (1989). Several possible events can compete to trigger the next transition, and each of these events has its own probabilistic distribution for determining the next state.

\subsection{Petri nets}

Petri nets Peterson (1981) define the structure of concurrent systems using a bipartite graph. One type of the graph's nodes, the places, represents the system states, and the second kind, the transitions, represents the net evolution Adi Mallikarjuna et al. (2007).

\subsection{Queuing networks}

Queuing networks are based on a customer-server paradigm, in which customers make service requests to the servers and these requests are queued at the server until they can be serviced. The arrival time for customers and the service time at a server are described as stochastic models. By defining the number of servers and the buffering capacity on each of them, we can determine performance metrics (including the number of customers in line, 
throughput-number of customers serviced per time unit, turnaround times, etc.). Different policies can be used (priorities; preemption; first in, first out; etc.) Li \& Li (2003).

\subsection{Calculus of communicating systems}

The formal language of calculus of communicating systems (CCS) provides primitives for concurrency and parallelism, based on synchronous communications between exactly two components. The language expressions are interpreted as a labeled transition system, and bi-simulation can be used to prove equivalence of models Hansson \& Jonsson (1990).

\subsection{Temporal logic}

Temporal logic is a system of rules and symbols used for representing propositions that can include the timing properties of the system Manna \& Pnueli (1992). It consists of a logic set of propositions that view time as a sequence of states and that can be true or false according to their state and their time of occurrence. Temporal logic has been used to verify formally timed automata. The idea is to check predictability of certain conditions according to the time that they occur, conditions that might eventually arise, or others that are guaranteed not to occur.

\subsection{Communicating sequential processes}

Communicating sequential processes (CSP) is a formal language based on process algebra that has been widely used to model concurrent systems Hoare (1985). Models are described using independent processes that interact with each other through message-passing representing the occurrence of events Wainer (2009).

\subsection{Specification and Description Language}

Specification and Description Language (SDL) was created to specify in a nonambiguous way the behavior of real-time applications. It was originally focused on communication systems, by providing a graphical and textual representation with equivalent semantics. A system is defined as a set of extended FSMs that can be interconnected Belina \& Hogrefe (1989).

\subsection{Event graphs}

Event graphs are oriented graphs that represent the organization of the events of a discrete event system Schruben et al. (2003). Events constitute nodes of the graph; that is, the vertices represent the state transition functions, and the links between nodes capture the scheduling of such events. Each link starts at the node performing the scheduling operation (which represents an event), and it ends at the node representing the event to be scheduled. Each scheduling relationship has an associated delay and condition (a Boolean function of the state), and an event is scheduled only when the condition is true Wainer (2009).

\subsection{Systems theory}

Systems-theoretical approaches derive from systems theory von Bertalanffy (1969). Systems theory represents every entity under study using the concept of system, which is seen as a collection of objects and their interactions. In systems theory, the system's global behavior is seen as a composition of the individual behavior of the components, and we can find emergent behavior that is not explicitly defined in the parts of the system. Systems theory is based on the idea that every phenomenon can be viewed as a mathematical relationship among a set of entities in the system. The theory is generic and tries to find common behavior and properties 
in different fields of study (for instance, hydraulics, economy, biology, or social sciences), thus providing a unified view of science and engineering.

\subsection{Discrete-Event Systems Specifications}

Discrete-Event Systems Specifications (DESS) formalism Zeigler $(1976 ; 1990)$ is a mathematical modeling technique derived from systems theory that allows the construction of hierarchical and modular models, providing a well-defined coupling of components. Given its hierarchical nature, DEVS allows the coupling of existing models modularly, allowing us to build complex systems from simple ones. DEVS theory provides a rigorous methodology for representing models, and it presents an abstract way of thinking about the world independently of the simulation mechanisms Wainer (2009).

\section{Phases in a Simulation Study}

There have been different kinds of life cycles proposed for studies in modeling and simulation. In this section, we summarize the basic steps that should be considered in doing a simulation study. The life cycle does not have to be interpreted as strictly sequential; it is iterative by nature, and sometimes transitions in opposite directions can appear. Likewise, some of the steps can be skipped, according to the complexity of the application. It is highly recommended to use a spiral cycle with incremental development for steps 2-8 (Section 5.2 through Section 5.8), which can cause a revision to earlier phases. Each phase in the spiral cycle should end with a working prototype including more functionality than the previous cycle:

\subsection{Problem formulation}

The simulation process begins with a practical problem that requires solving or understanding. It might be the case of a cargo company trying to develop a new strategy for truck dispatching or an astronomer trying to understand how a nebula is formed. At this stage, we must understand the behavior of the system of interest (which can be a natural or artificial system, existing or not), organizing the system's operation as objects and activities within the experimental framework of interest. Then we need to analyze different alternatives of solutions by investigating other previously existing results for similar problems. The most acceptable solution should be chosen (omitting this stage could cause the selection of an expensive or wrong solution). We also must identify the input/ output variables and classify them into decision variables (controllable) or parameters (non-controllable). If the problem involves performance analysis, this is the point at which we can also define performance metrics (based on the output variables) and an objective function (i.e., a combination of some of the metrics). At this stage, we can also do risk analysis and decide whether to follow or discard the project.

\subsection{The Conceptual Model}

This step consists of building a high-level description of the structure and behavior of the system and identifying all the objects with their attributes and interfaces. We also must define what the state variables are, how they are related, and which ones are important for the study. In this step, key aspects of the requirements are expressed (if possible, using a formalism, which introduces a higher degree of precision). During the definition of the conceptual model, we need to reveal features that are of critical significance (e.g., possibility of instability, deadlock, or starvation). We must also document nonfunctional information-for instance, possible future changes, nonintuitive (or non-formal) behavior, and the relation with the environment. 


\subsection{The collection and analysis of input/output data phase}

In this phase, we must study the system to obtain input/output data. To do so, we must observe and collect the attributes chosen in the previous phase. When the system entities are studied, we try to associate them with a timing value. Another important issue during this phase is the selection of a sample size that is statistically valid and a data format that can be processed with a computer. Finally, we must decide which attributes are stochastic and which are deterministic. In some cases, there are no data sources to collect (for instance, for nonexisting systems). In those cases, we need to try to obtain data sets from similar systems (if available). Another option is to use a stochastic approach to provide the data needed through random number generation.

\subsection{Modeling phase}

In the modeling phase, we must build a detailed representation of the system based on the conceptual model and the I/O data collected. The model is built by defining objects, attributes, and methods using a chosen paradigm. At this point, a specification model is created, including the set of equations defining its behavior and structure. After finishing this definition, we must try to build a preliminary structure of the model (possibly relating the system variables and performance metrics), carefully describing any assumptions and simplifications and collecting them into the model's EF.

\subsection{Simulation phase}

During the simulation stage, we must choose a mechanism to implement the model (in most cases using a computer and adequate programming languages and tools), and a simulation model is constructed. During this step, it might be necessary to define simulation algorithms and to translate them into a computer program. In this phase, we also must build a model of the EF for the simulation.

\subsection{Verification and validation}

During the previous steps, three different models are built: the conceptual model (specification), the system's model (design), and the simulation model (executable program). We need to verify and validate these models. Verification is related to the internal consistency among the three models (is the model correctly implemented?). Validation is focused on the correspondence between model and reality: are the simulation results consistent with the system being analyzed? Did we build the right model? Based on the results obtained during this phase, the model and its implementation might need refinement. As we will discuss in the next section, the $\mathrm{V} \& \mathrm{~V}$ process does not constitute a particular phase of the life cycle, but it is an integral part of it. This process must be formal and must be documented correctly because later versions of the model will require another round of $V \& V$, which is, in fact, one of the most expensive phases in the cycle.

\subsection{Experimentation}

We must execute the simulation model, following the goals stated in the conceptual model. During this phase, we must evaluate the outputs of the simulator, using statistical correlation to determine a precision level for the performance metrics. This phase starts with the design of the experiments, using different techniques. Some of these techniques include sensitivity analysis, optimization, variance reduction (to optimize the results from a statistical point of view), and ranking and selection (comparison with alternative systems). 


\subsection{Output analysis phase}

In the output analysis phase, the simulation outputs are analyzed in order to understand the system behavior. These outputs are used to obtain responses about the behavior of the original system. At this stage, visualization tools can be used to help with the process. The goal of visualization is to provide a deeper understanding of the real systems being investigated and to help in exploring the large set of numerical data produced by the simulation.

\section{M\&S Tools and Environments for WSNs}

Different schemes developed for Wireless Sensor Networks (WSNs) are verified by analytical techniques, simulations, and test bedsCinque et al. (2007); Lopez et al. (2005). Analytical modeling may provide quick insight but fail to give realistic results for reasons like limited energy, memory, and processing power, and sheer number, unattended operation, harsh environments of sensor nodes Chen et al. (2006). Real world implementation and test beds are the most accurate ways to study WSNs but such methods requires huge effort, time and money. Simulations provide a good approximation to verify different schemes and applications developed for WSNs at low cost and in less time. To have credible results through simulations, correct modeling and the selection of simulation tool plays a vital role. In the following sections, we present brief description of different models as well as simulation tools available to study WSNs.

\subsection{Simulation Tools and Environments}

\subsubsection{NS-2}

NS- $2^{5}$ is a discrete event, object oriented, general purpose network simulator written in C++. It is the most widely used simulator Kurkowski et al. (2005). Its main focus is IP networks. To simulate WSNs with more or less 100 nodes, NS-2 can be a good choice because of its large community but for $100+$ nodes, it is no more scalable Naoumov \& Gross (2003). "One of the problems of ns2 is its object-oriented design that introduces much unnecessary inter-dependence between modules. Such interdependence sometimes makes the addition of new protocol models extremely difficult, which can only be mastered by those who have intimate familiarity with the simulator. "Chen et al. (2006). SensorSim Park et al. (2000) is a NS-2 based simulator for modeling sensor networks. Some WSN specific features are included but because of the "unfinished nature of the software $^{\prime \prime}$, the simulator is no longer available.

\subsubsection{OMNeT++}

OMNeT ++ Varga (2001) is a discrete event, component based, general purpose, public source, modular simulation framework written in $\mathrm{C}++$. It provides a strong GUI support for animation and debugging. Mobility framework (MF) Drytkiewicz et al. (2003) for OMNet++ is specific purpose add-on to simulate ad-hoc networks. The lack of a WSN specific module library Lopez et al. (2006) may be a problem currently but many research groups are working to add WSN specific additional modules. SenSim Mallanda et al. (2005) is OMNeT based simulation framework for WSN. It provides the basic implementation of different hardware (e.g., basic radio, and CPU) and software (simple routing schemes) modules for WSN. It provides a template with basic implementation or empty body which can help anyone to jump start simulating WSNs Egea-Lopez et al. (2006).

\footnotetext{
5 www.isi.edu/nsnam/ns

${ }^{6}$ nesl.ee.ucla.edu/projects/sensorsim
} 


\subsubsection{NesCT}

$\mathrm{NesCT}^{7}$ is an add-on for OMNeT++ which allows simulation of TinyOS based sensor networks in OMNeT (language translator between OMNeT and TinyOS implementations).

\subsubsection{PAWiS}

$\mathrm{PAWiS}^{8}$ simulation framework is OMNeT plus plus based discrete event simulation framework. It provides a rich library of modules and supports mobility and environmental dynamics. It also provides a simulation template for users to quick start simulation study Madani et al. (2008); Weber et al. (2007).

\subsubsection{GlomoSim}

Global Mobile Information System Simulator (GlomoSim) Zeng et al. (1998) is a library based general purpose, parallel simulator written in Parsec ${ }^{9}$. It can simulate up to 10,000 nodes Lopez et al. (2005) and can be very useful in studying large scale WSNs. . GlomoSim is superseded by QualNet ${ }^{10}$, a commercial network simulator. sQualNet Varshney et al. (2007), an evaluation framework for sensor networks, based on QualNet is released recently.

\subsubsection{OPNET}

OPNET Modeler ${ }^{11}$ is general purpose, object oriented, C-based discrete event simulation environment. It's commercial and therefore not used widely. It comes with a version for academic use but with limited capabilities. OPNET is a large and powerful software with a wide variety of possibilities. OPNET can be used as a research tool and also as a network design/analysis tool. OPNET was originally built for the simulation of fixed networks, and therefore, it contains extensive libraries of accurate models from commercially available fixed network hardware and protocols Cai \& Jia (2009); Hammoodi et al. (2009); Hasan et al. (2009); Zhuo et al. (2007).

\subsubsection{SENSE}

SENSE Chen et al. (2006) is a sensor network specific, component based simulator written in $\mathrm{C}++$ built on the top of COST Chen \& Szymanski (2002). Parallel simulation can be done to study large scale WSNs. It provides basic implementations like AODV and DSR as well as oversimplified models for batter and energy consumption.

\subsubsection{Ptolemy II and J-Sim}

Ptolemy II Liu et al. (2001) and J-Sim Miller et al. (1997); Sobeih et al. (2005; 2006) are general purpose, component based simulation frameworks written Java. Both simulation frameworks provide a rich support for WSNs.

\subsubsection{Cell-DEVS}

The Cell-DEVS formalism allows defining cellular models based on the discrete-event system specification. Cell-DEVS allows defining asynchronous cell spaces with explicit timing definition. This approach is still based on the formal specifications of DEVS, but it allows the user to

\footnotetext{
${ }^{7}$ www.omnetpp.org

8 www.ict.tuwien.ac.at/pawis

${ }^{9}$ pcl.cs.ucla.edu/projects/parsec

10 www.scalable-networks.com

11 www.opnet.com
} 
focus on the problem to be solved by using simple rules for modeling (like with CA). Explicit timing delay constructions can be used to define precise timing in each cell. This approach allows enhancing the modeling experience in different aspects. In terms of performance, only active cells execute their local computing function, and the execution results are spread out after a predefined delay (only if a state change has occurred). The delay function provides a natural mechanism for defining timing information.

The modeling technique permits keeping the ability of CA to describe complex systems using very simple rules, while also permitting us to bridge the gap between a discrete time and a discrete event description like DEVS. The use of DEVS as the basic formal specification mechanism enables us to define interactions with models defined in other formalisms. Individual cells can provide data to those models; integration between them could enable defining of complex hybrid systems and multimodels developed with different techniques and integrated through a DEVS interface. This approach provides "evolvability" of the models through a technique that is easy to understand and to map into other existing techniques, while having the potential of evolving into complex models Qela et al. (2009).

\subsubsection{GTNetS}

The "Georgia Tech Network Simulator," (GTNetS) ${ }^{12}$ developed and maintained by the Department of Electrical and Computer Engineering at Georgia Tech is an object-oriented design written completely in $\mathrm{C}++$. The design of GTNetS matches closely the design of actual network protocol stacks and other network elements. Further, GTNetS was designed from the beginning to run a distributed environment, leading to better scalability Cheng et al. (2006); Riley (2003); Zhang \& Riley (2004).

\subsubsection{SystemC}

SystemC is a $\mathrm{C}++$ based modeling platform supporting design abstractions at the registertransfer, behavioral, and system levels. The SystemC classes add the necessary constructs to $\mathrm{C}++$ for modeling systems and hardware at various levels of abstraction-from the abstract untimed models to cycle-accurate RTL models. The power of SystemC is that it can be used as a common language by system designers, software engineers, and hardware designers Rafiee et al. (2009); Vasilevski et al. (2007).

\subsubsection{Prowler}

Prowler ${ }^{13}$ is an event-driven wireless network simulator designed to run in Matlab environment. The simulator, written originally to simulate Berkeley MICA motes, is extendable also for more general platforms. Prowler is implemented in Matlab language ( $\mathrm{m}$-file) which makes direct simulation code, e.g., routing protocol or application, interchange between simulator and sensor platforms impossible. Benefits gained from Matlab environment are easy prototyping of applications, integration of different optimization algorithms, GUI interface and good visualization capabilities.

\subsubsection{NCTUns2.0}

NCTUns2. $0^{14}$ is a discrete event simulator whose engine is embedded in the kernel of a UNIX machine. The actual network layer packets are tunnelled through virtual interfaces that simu-

\footnotetext{
12 http://www.ece.gatech.edu/research/labs/MANIACS/GTNetS/

13 http://www.isis.vanderbilt.edu/projects/nest/prowler

14 http://nsl.csie.nctu.edu.tw
} 
late lower layers and physical devices. This notable feature allows simulations to be fed with real program data sources. A useful GUI is available in addition to a high number of protocols and network devices, including wireless LAN. Unfortunately, no specific designs for WSN are included.

\subsubsection{JiST/SWANS}

JiST/SWANS ${ }^{15}$ is a discrete event simulation framework that embeds the simulation engine in the Java bytecode. Models are implemented in Java and compiled. Then, bytecodes are rewritten to introduce simulation semantics. Afterwards, they are executed on a standard JVM. This implementation allows the use of unmodified existing Java software in the simulation, as occurs with NCTUns2.0 and UNIX programs.

\subsubsection{SSFNet}

SSFNet ${ }^{16}$ is a set of Java network models built over the Scalable Simulation Framework (SSF). SSF is a specification of a common API for simulation, that assures portability between compliant simulators. There are multiple Java and $\mathrm{C}++$ implementations of SSF. DartmouthSSF (DaSSF) [30], for instance, is a C++ implementation of SSF oriented to (parallel) simulation of very large scale communication networks.

\subsubsection{Ptolemy II}

Ptolemy II ${ }^{17}$ is a set of Java packages that support different models of simulation paradigms (e.g. continuos time, dataflow, discrete-event). It also addresses the modeling, simulation and design of concurrent, real-time and embedded systems.

\subsection{Emulation Tools}

In addition to the above cited simulation tools/environments for WSNs, there are number of emulation tools/environments as well. Some of the these are TOSSIM Levis et al. (2003), EmStar Girod et al. (2004), and ATEMU Polley et al. (2004). Such tools come with inherent advantage. Whatever code is used for simulation/emulation, the same code is used on the real sensor node with slight modifications. It also provides detailed information about resource utilization. The main problem with such frameworks is "the user is tied to a single platform either software or hardware (typically MICA motes), and to a single programming Language (typically TinyOS/NesC)"Lopez et al. (2005).

\subsection{Other Tools}

There are many tools which do not fall into either simulation or emulation catagories like TEPAWSN Man et al. (2009) which is a tool environment for Wireless Sensor Networks.

\section{Summary}

Wireless Sensor Netwroks is an emerging field with many apllication in almost all walk of life. Researchers are actively involved in the development of new and improving the existing techiques and technologies for making the life more easier. Each of these developed and/or improved techniques need to be extensively testes and varified before it can be used in the

\footnotetext{
15 http://jist.ece.cornell.edu/

16 http://www.ssfnet.org

17 http:/ / ptolemy.berkeley.edu/ptolemyII/
} 
actual production. Most accurate and reliable method is, of-course, the real-world implementation and test-beds. But these are sometimes not possible or even harder. In that case the method of the choice is the modeling and simulation.

In this chapter we presented a partial list of available models and simulation/emulation tools for the wireless sensor networks, available. The chapter started with an introduction and the historical perspective to the field of WSN and some of its applications. We then briefly discussed the different discrete event modeling and simulation methodologies after which the major steps/phases in a M\&S study are outlines with the identification of major milestone. The chapter concludes with a list of different models, simulation and emulation tools.

\section{References}

Abramson, N. (1970). The ALOHA System-Another alternative for computer communications, Fall Joint Computing Conf, pp. 281-285.

Adi Mallikarjuna, R. V., Kumar, A. V. U. \& Janakiram, D. (2007). e-petri net model for programming integrated network of wireless sensor networks and grids, Proc. 7th IEEE Int. Conf. Computer and Information Technology CIT 2007, pp. 1038-1043.

Akyildiz, I., Su, W., Sankarasubramaniam, Y. \& Cayirci, E. (2002). A survey on sensor networks, IEEE Communications Magazine 40(8): 102-114.

Alur, R. \& Dill, D. L. (1994). A theory of timed automata, Theor. Comput. Sci. 126(2): 183-235.

Antoine-Santoni, T., Santucci, J. F., de Gentili, E. \& Costa, B. (2006). Using wireless sensor network for wildfire detection. a discrete event approach of environmental monitoring tool, Proc. First international Symp. Environment Identities and Mediterranean Area ISEIMA '06, pp. 115-120.

Banks, J., J. S. C. B. L. N. \& Nicol., D. (2005). Discrete-event system simulation, 4th ed., Upper Saddle River, NJ: Prentice Hall.

Belina, F. \& Hogrefe, D. (1989). The ccitt-specification and description language sdl, Comput. Netw. ISDN Syst. 16(4): 311-341.

Cai, J. \& Jia, B. (2009). Network simulation based on opnte and application, Proc. First Int. Workshop Education Technology and Computer Science ETCS '09, Vol. 1, pp. 199-202.

Callaway, E., Paul Gorday, L. H., Guiterrez, J. A., M. Naeve, B. H. \& Bahl, V. (2002). Home Networking with IEEE802.15.4: A Developing Standard for Low-Rate Wireless Personal Area Networks, IEEE Communication Magazine 40(8).

Cassandras, C. G. (1993). Discrete event systems: Modeling and performance analysis, Homewood, IL:Aksen: Irwin.

Cellier, F. E. \& Kofman., E. (2006). Continuous system simulation, Springer Science+Business Media.

Cerpa, A., Elson, J., Estrin, D., Girod, L., Hamilton, M. \& Zhao, J. (2001). Habitat monitoring: Application driver for wireless communications technology, In Proceedings of the 2001 ACM SIGCOMM Workshop on Data Communications in Latin America and the Caribbean.

Chen, G., Branch, J., Pflug, M., Zhu, L. \& Szymanski2, B. (2006). Advances in Pervasive Computing and Networking, Springer US, chapter Sense: A Wireless Sensor Network Simulator, pp. 249-267.

Chen, G. \& Szymanski, B. (2002). COST: a component-oriented discrete event simulator, Proceedings of the Winter Simulation Conference.

Cheng, L., Zhang, X. \& Bourgeois, A. G. (2006). Ieee 802.15.4 simulation module in network simulator gtnets, Proc. VTC 2006-Spring Vehicular Technology Conf. IEEE 63rd, Vol. 3, pp. 1308-1312. 
Cinque, M., Cotroneo, D., Di Martinio, C. \& Russo, S. (2007). Modeling and assessing the dependability ofwireless sensor networks, Proc. 26th IEEE Int. Symp. Reliable Distributed Systems SRDS 2007, pp. 33-44.

Colitti, W., Steenhaut, K., Lemmens, B. \& Borms, J. (2009). Simulation tool for wireless sensor network constellations in space, Proc. Int. Conf. Ultra Modern Telecommunications $\mathcal{E}$ Workshops ICUMT'09, pp. 1-5.

Demo, J., Steiner, A., Friedersdorf, F. \& Putic, M. (2010). Development of a wireless miniaturized smart sensor network for aircraft corrosion monitoring, Proc. IEEE Aerospace Conf, pp. 1-9.

Dietrich, D., Loy, D. \& Schweinzer, H. (2001). Open Control Networks LonWorks/EIA 709 Technology. Kluwer Academic Publishers.

Drytkiewicz, W., Sroka, S., Handziski, V., Koepke, A. \& Karl, H. (2003). A Mobility Framework for OMNeT++, 3rd International OMNeT++ Workshop.

Egea-Lopez, E., Ponce-Marin, F. \& Vales-Alonso, J. (2006). Obiwan: wireless sensor networks with omnet++, Proc. IEEE Mediterranean Electrotechnical Conf. MELECON 2006, pp. 777-780.

Fishwick, P. A. (1995). Simulation model design and execution: Building digital worlds, Englewood Cliffs, NJ: Prentice Hall.

Fu, S., Hou, Z.-G. \& Yang, G. (2009). An indoor navigation system for autonomous mobile robot using wireless sensor network, Proc. Int. Conf. Networking, Sensing and Control ICNSC '09, pp. 227-232.

Girod, L., Stathopoulos, T., Ramanathan, N., Elson, J., Estrin, D., Osterweil, E. \& Schoellhammer, T. (2004). A system for simulation, emulation, and deployment of heterogeneous sensor networks, 2nd international Conference on Embedded Networked Sensor Systems.

Glynn, P. (1989). A gsmp formalism for discrete event systems, Proceedings of the IEEE 77(1): 14 $-23$.

Hammoodi, I. S., Stewart, B. G., Kocian, A. \& McMeekin, S. G. (2009). A comprehensive performance study of opnet modeler for zigbee wireless sensor networks, Proc. Third Int. Conf. Next Generation Mobile Applications, Services and Technologies NGMAST '09, pp. 357-362.

Hansson, H. \& Jonsson, B. (1990). A calculus for communicating systems with time and probabilities, Proc. th Real-Time Systems Symp., pp. 278-287.

Hasan, M. S., Yu, H., Carrington, A. \& Yang, T. C. (2009). Co-simulation of wireless networked control systems over mobile ad hoc network using simulink and opnet, IET Communications 3(8): 1297-1310.

Hoare, C. A. R. (1985). Communicating sequential processes, Englewood Cliffs, NJ: Prentice Hall International.

$\mathrm{Hu}$, J. (2008). The application of wireless sensor networks to in-service motor monitoring and energy management, Proc. First Int. Conf. Intelligent Networks and Intelligent Systems ICINIS '08, pp. 165-169.

Isreb, M. (2006). Parallel distributed wireless sensor network application for critical infrastructure risk analysis of fossil fuelled power stations, Proc. IEEE Int Information Acquisition Conf, pp. 301-305.

Jubin, J. \& Tornow, J. D. (1987). The DARPA Packet Radio Network Protocols, Proceedings of the IEEE, Vol. 75, pp. 21-32. 
Ke, Z., Yang, L., Wang-hui, X. \& Heejong, S. (2008). The application of a wireless sensor network design based on zigbee in petrochemical industry field, Proc. First Int. Conf. Intelligent Networks and Intelligent Systems ICINIS '08, pp. 284-287.

Kurkowski, S., Camp, T. \& Colagrosso, M. (2005). MANET simulation studies: the incredibles, Mobile Computing and Communications Review 9(4): 50-61.

Law, A. M. \& Kelton, W. D. (2000). Simulation modeling and analysis, 3rd ed, Boston: McGraw Hill.

Lee, S. H., Lee, S., Song, H. \& Lee, H. S. (2009). Wireless sensor network design for tactical military applications : Remote large-scale environments, Proc. IEEE Military Communications Conf. MILCOM 2009, pp. 1-7.

Levis, P., N.Lee, Welsh, M. \& D.Culler (2003). TOSSIM: accurate and scalable simulation of entire tinyOS applications, 1st international Conference on Embedded Networked Sensor Systems, pp. 126-137.

URL: $h t t p: / / w w w . c s . b e r k e l e y . e d u / \tilde{p} a l / p u b s /$ nido.pdf

Li, G.-L. \& Li, V. O. K. (2003). Networks of queues: myth and reality, Proc. IEEE 18th Annual Workshop Computer Communications CCW 2003, pp. 154-158.

Liu, H., Liu, X. \& Lee, E. (2001). Modeling distributed hybrid systems in Ptolemy II, American Control Conference, Vol. 6.

Lopez, E., Alonso, V., Sala, M., Mario, P. \& Haro, G. (2006). Simulation scalability issues in wireless sensor networks, IEEE Communications Magazine 44(7): 64-73.

Lopez, E. E., Alonso, J. V., Sala, A. M., Marino, P. P. \& Haro, J. G. (2005). Simulation tools for wireless sensor networks, International Symposium on Performance Evaluation of Computer and Telecommunication Systems (SPECTS05), Philadelphia, USA.

URL: http://ait.upct.es/ẽegea/

Madani, S. A., Weber, D. \& Mahlknecht, S. (2008). Tpr: Dead end aware table less position based routing scheme for low power data-centric wireless sensor networks, Proc. Int. Symp. Industrial Embedded Systems SIES 2008, pp. 149-154.

Mallanda, C., Suri, A., Kunchakarra, V., Iyengar, S., Kannan, R. \& Durresi, A. (2005). Simulating Wireless Sensor Networks with Omnet++, [submitted to IEEE Computers].

URL: http://csc.lsu.edu/iyengar/final-papers/SensorSimulator-IEEE-Computers.pdf

Man, K. L., Vallee, T., Leung, H. L., Mercaldi, M., van der Wulp, J., Donno, M. \& Pastrnak, M. (2009). Tepawsn - a tool environment for wireless sensor networks, Proc. 4th IEEE Conf. Industrial Electronics and Applications ICIEA 2009, pp. 730-733.

Manna, Z. \& Pnueli, A. (1992). The temporal logic of reactive and concurrent systems, New York:Springer Verlag.

Miller, J., Nair, R., Zhang, Z. \& Zhao, H. (1997). JSIM: A Java-based simulation and animation environment, 30th Annual Simulation Symposium, pp. 31-42.

Molina-Garcia, A., Fuentes, J. A., Gomez-Lazaro, E., Bonastre, A., Campelo, J. C. \& Serrano, J. J. (2007). Application of wireless sensor network to direct load control in residential areas, Proc. IEEE Int. Symp. Industrial Electronics ISIE 2007, pp. 1974-1979.

Naoumov, V. \& Gross, T. (2003). Simulation of large ad hoc networks, 6th ACM international Workshop on Modeling Analysis and Simulation of Wireless and Mobile Systems, San Diego, CA, USA.

URL: http://citeseer.ist.psu.edu/naoumov03simulation.html

Park, S., Savvides, A. \& Srivastava, M. B. (2000). SensorSim: a simulation framework for sensor networks, International Workshop on Modeling Analysis and Simulation of Wireless 
and Mobile Systems.

URL: $h t t p: / / p o r t a l . a c m . o r g / c i t a t i o n . c f m ? i d=346870$

Peterson, J. L. (1981). Petri net theory and the modeling of systems, Englewood Cliffs, NJ: Prentice Hall.

Polley, J., Blazakis, D., McGee, J., Rusk, D. \& Baras, J. (2004). ATEMU: a fine-grained sensor network simulator, First Annual IEEE Communications Society Conference on Sensor and Ad Hoc Communications and Networks (SECON), pp. 145-152.

Qela, B., Wainer, G. \& Mouftah, H. (2009). Simulation of large wireless sensor networks using cell-devs, Proc. Winter Simulation Conf. (WSC) the 2009, pp. 3189-3200.

Rafiee, M., Ghaznavi-Ghoushchi, M. B., Kheiri, S. \& Seyfe, B. (2009). Modeling and simulation of wireless sensor network (wsn) with specc and systemc, Proc. Int. Conf. Computer Engineering and Technology ICCET '08, Vol. 1, pp. 515-519.

Raghavendra, C., Sivalingam, K. M. \& Znati, T. (2004). Wireless Sensor Networks, Kluwer Academic Publishers.

Riley, G. F. (2003). Large-scale network simulations with gtnets, Proc. Winter Simulation Conf, Vol. 1, pp. 676-684.

Sarkar, T. K., Oliner, M. R., A., A., Salazar-Palma, Magdalena, Sengupta \& L., D. (2006). History of Wireless, Vol. 1, 1 edn, Wiley Series in Microwave and Optical Engineering, chapter 7, pp. 451-452.

Sauter, T., Dietrich, D. \& Kastner, W. (2002). EIB: Installation Bus System, Wiley-VCH.

Schruben, L. W., Roeder, T. M., Chan, W. K., Hyden, P. \& Freimer, M. (2003). Advanced event scheduling methodology: advanced event scheduling methodology, WSC '03: Proceedings of the 35th conference on Winter simulation, Winter Simulation Conference, pp. 159-165.

Sobeih, A., Chen, W.-P., Hou, J. C., Kung, L.-C., Li, N., Lim, H., Tyan, H.-Y. \& Zhang, H. (2005). J-sim: a simulation environment for wireless sensor networks, Proc. 38th Annual Simulation Symp, pp. 175-187.

Sobeih, A., Hou, J. C., Kung, L.-C., Li, N., Zhang, H., Chen, W.-P., Tyan, H.-Y. \& Lim, H. (2006). J-sim: a simulation and emulation environment for wireless sensor networks, 13(4): 104-119.

Taylor, M. (1996). Partial differential equations: Basic theory, Springer Verlag.

Toffoli, T. \& Margolus., N. (1987). Cellular automata machines: A new environment for modeling, Cambridge, MA: MIT Press.

Toh, S.-H., Lee, S.-C. \& Chung, W.-Y. (2008). Wsn based personal mobile physiological monitoring and management system for chronic disease, Proc. Third Int. Conf. Convergence and Hybrid Information Technology ICCIT '08, Vol. 1, pp. 467-472.

Varga, A. (2001). 'The OMNeT++ Discrete Event Simulation System, In the Proceedings of the European Simulation Multiconference.

URL: $h$ ttp://www.omnetpp.org/links.php?category=Publications

Varshney, M., Xu, D., Srivastava, M. B. \& Bagrodia, R. L. (2007). sQualNet: An Accurate and Scalable Evaluation Framework for Sensor Networks, International Conference on Information Processing in Sensor Networks.

URL: $h t t p: / / p c l . c s . u c l a . e d u / p a p e r s / f i l e s / s p o t s 07-v a r s h n e y . p d f$

Vasilevski, M., Pecheux, F., Aboushady, H. \& de Lamarre, L. (2007). Modeling heterogeneous systems using systemc-ams case study: A wireless sensor network node, Proc. IEEE Int. Behavioral Modeling and Simulation Workshop BMAS 2007, pp. 11-16. 
von Bertalanffy, L. (1969). General system theory: Foundations, development, applications, New York: G. Braziller.

Wainer, G. A. (2009). Discrete-Event Modeling and Simulation A Practitioner's Approach, CRC Press.

Weber, D., Glaser, J. \& Mahlknecht, S. (2007). Discrete event simulation framework for power aware wireless sensor networks, Proc. 5th IEEE Int Industrial Informatics Conf, Vol. 1, pp. 335-340.

Zeigler, B. P. (1976). Theory of modeling and simulation, New York: Wiley Interscience.

Zeigler, B. P. (1990). Object-oriented simulation with hierarchical, modular models: Intelligent agents and endomorphic systems, Boston: Academic Press.

Zeng, X., Bagrodia, R. \& Gerla, M. (1998). GloMoSim: a library for parallel simulation of largescale wireless networks, Twelfth Workshop on Parallel and Distributed Simulation.

URL: $h t t p: / / q u a l n e t . c o m / p d f / g l o m o s i m . p d f$

Zhang, X. \& Riley, G. F. (2004). Bluetooth simulations for wireless sensor networks using gtnets, Proc. IEEE Computer Society's 12th Annual Int. Symp. Modeling, Analysis, and Simulation of Computer and Telecommunications Systems (MASCOTS 2004), pp. 375-382.

Zhao, F. \& Guibas, L. (2004). Wireless Sensor Networks, an information processing approach, Morgan Kaufmann. 294-300.

Zhuo, Z., Donglei, X., Qianli, L. \& Maopei, L. (2007). Wireless modeling of hf networks in opnet, Proc. Int Microwave, Antenna, Propagation and EMC Technologies for Wireless Communications Symp, pp. 285-288. 
Discrete Event

Simulations

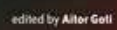

\section{Discrete Event Simulations}

Edited by Aitor Goti

ISBN 978-953-307-115-2

Hard cover, 330 pages

Publisher Sciyo

Published online 18, August, 2010

Published in print edition August, 2010

Considered by many authors as a technique for modelling stochastic, dynamic and discretely evolving systems, this technique has gained widespread acceptance among the practitioners who want to represent and improve complex systems. Since DES is a technique applied in incredibly different areas, this book reflects many different points of view about DES, thus, all authors describe how it is understood and applied within their context of work, providing an extensive understanding of what DES is. It can be said that the name of the book itself reflects the plurality that these points of view represent. The book embraces a number of topics covering theory, methods and applications to a wide range of sectors and problem areas that have been categorised into five groups. As well as the previously explained variety of points of view concerning DES, there is one additional thing to remark about this book: its richness when talking about actual data or actual data based analysis. When most academic areas are lacking application cases, roughly the half part of the chapters included in this book deal with actual problems or at least are based on actual data. Thus, the editor firmly believes that this book will be interesting for both beginners and practitioners in the area of DES.

\section{How to reference}

In order to correctly reference this scholarly work, feel free to copy and paste the following:

Stefan Mahlknecht, Sajjad A. Madani and Jawad Kazmi (2010). Wireless Sensor Networks: Modelling and Simulation, Discrete Event Simulations, Aitor Goti (Ed.), ISBN: 978-953-307-115-2, InTech, Available from: http://www.intechopen.com/books/discrete-event-simulations/wireless-sensor-networks-modelling-andsimulation

\section{INTECH}

open science | open minds

\section{InTech Europe}

University Campus STeP Ri

Slavka Krautzeka 83/A

51000 Rijeka, Croatia

Phone: +385 (51) 770447

Fax: +385 (51) 686166

www.intechopen.com

\section{InTech China}

Unit 405, Office Block, Hotel Equatorial Shanghai

No.65, Yan An Road (West), Shanghai, 200040, China

中国上海市延安西路65号上海国际贵都大饭店办公楼405单元

Phone: +86-21-62489820

Fax: $+86-21-62489821$ 
(C) 2010 The Author(s). Licensee IntechOpen. This chapter is distributed under the terms of the Creative Commons Attribution-NonCommercialShareAlike-3.0 License, which permits use, distribution and reproduction for non-commercial purposes, provided the original is properly cited and derivative works building on this content are distributed under the same license. 